\title{
A LLAVE DE NUEVA ESPAÑA. PROYECTOS DEFENSIVOS PARA LOS TERRITORIOS DE LUISIANA (1770-1795) ${ }^{1}$
}

PEDRO CRUZ FREIRE

Departamento de Historia del Arte. Universidad de Sevilla pedrocruz@us.es

\begin{abstract}
Resumen: El presente estudio trata de analizar, a través de diferentes propuestas, el estado de las fortificaciones de la provincia de Luisiana y las líneas de actuación que para su defensa se llevaron a cabo en aquel territorio en el periodo comprendido entre 1764 y 1795. Para ello, han sido recopilados los diversos informes elaborados por el militar Francisco Bouligny y los ingenieros Francisco Sabatini y Juan María Perchet, entre otros, documentos que han ayudado a reconstruir las principales cuestiones defensivas y estratégicas de la zona.
\end{abstract}

Palabras clave: Nueva Orleans / Ingeniería Militar / fortificaciones / río Misisipi.

\section{THE KEY OF NUEVA ESPAÑA. DEFENSIVE PROJECTS FOR LUISIANA'S TERRITORIES (1770-1795)}

Abstract: The present study intends to analyze, through different proposals, the condition of fortifications in Louisiana and defensive procedures accomplished in that territory between 1764-1795. Thus, various reports performed by Francisco Bouligny, Francisco Sabatini and Juan María Perchet, among others, have helped to rebuilt defensive and strategic issues of this area.

Key Words: New Orleans / Military engineering / fortifications / Mississippi.

En 1763, una vez consumada la Guerra de los Siete Años y en virtud de la Paz de París, Francia modificaba el panorama geoestratégico americano con la cesión de los territorios de Luisiana a España, hecho rubricado en el Tratado de Fontainebleau. ${ }^{2}$

Mediante este pacto, el país galo maquillaba la desastrosa participación española en el conflicto y aliviaba las consecuencias de la obligada permuta ejecutada con los británicos entre las ciudades de La Habana y Manila por los territorios hispanos en la Florida. ${ }^{3}$ Como consecuencia de ello, Luisiana ocuparía un lugar de elevado interés para el entramado comercial y defensivo español establecido en el entorno del seno mexicano. Sin embargo, este intercambio trajo consigo una inversión notable, no solo en términos económicos, sino también en la asimilación de una serie de nuevas responsabilidades que garantizasen el correcto funcionamiento de la nueva provincia. Entre ellas, asimilar la gobernación de unos territorios administrados por manos extranjeras durante casi me-

* Fecha de recepción: 15 de enero de 2018 / Fecha de aceptación: 26 de febrero de 2018.

1 El presente trabajo se enmarca dentro del proyecto de investigación HAR 2015-63805-P "Ingenieros Militares en el Caribe y el Golfo de México Durante el Siglo XVIII. Diálogo Cultural, Circulación Transnacional y Conflictos Globales".

2 DEL CANTILLO, Alejandro, Tratados, convenios y declaraciones de Paz y de Comercio que han hecho con las potencias extranjeras los monarcas españoles de la casa de Borbón desde el año 1700 hasta el día. Madrid, imprenta de Alegría y Charlain, 1843, p. 485.

3 SAN MIGUEL PÉREZ, Enrique, "La crisis de la estrategia política española en Norteamérica: de Bernardo de Gálvez a Luis de Onís" en MARTíNEZ PEÑAS, Leandro y FERNÁNDEZ RODRÍGUEZ, Manuela (Coords.), El ejército y la armada en el noroeste de América: Nootka y su tiempo. Madrid, Universidad Rey Juan Carlos, 2012, p. 30. 
dio siglo, desarrollar una nueva política de poblamiento, emigración y desarrollo financiero y desplegar un renovado planteamiento defensivo acorde a las nuevas exigencias bélicas y las posibilidades geoestratégicas que ofrecía el paraje. Con relación a esta última, se ha creído conveniente la redacción de este estudio, con el fin de valorar el ingente esfuerzo llevado a cabo por la Corona española para salvaguardar un territorio de suma importancia para los intereses de la nación y el control territorial de una zona tan delicada y conflictiva como fue el entorno caribeño. Para ello, se han analizado varios informes realizados por ingenieros y personalidades ligadas al entorno militar que describen la situación defensiva de Luisiana, examinan nuevos métodos de protección y proponen nuevos proyectos de remodelación o construcción de fortificaciones. De este modo, su estudio revela nuevas claves para comprender los principales procedimientos defensivos aplicados a este territorio en el último tercio de la centuria.

Luisiana era una región de difícil protección dada su extensa geografía y la imposibilidad de controlarla totalmente, tanto por falta de recursos financieros como de personal humano. Así pues, dentro de este inabarcable territorio, el río Misisipi jugaba un papel fundamental articulando a lo largo de su cuenca la mayor parte de la actividad vital, comercial y defensiva. Por ello, no es de extrañar que los principales núcleos poblaciones se instalasen en las inmediaciones de su ribera, destacando entre todas ellas, la ciudad de Nueva Orleans.

Si bien el traspaso de poderes entre Francia y España se formuló de manera correcta y sin ningún tipo de incidencias, la corona no pudo evitar un espíritu de resistencia entre los habitantes de Nueva Orleans, quienes no compartían la decisión de su monarca. En 1768, tan solo cinco años después de lo estipulado en el tratado de Fontainebleau, una partida de insurrectos colonos se rebeló con el objetivo de expulsar al por entonces gobernador don Antonio de Ulloa, cuyo mandato solo abarcó dos años. ${ }^{4}$ Bajo órdenes de pacificar y recuperar su control, fue enviado el militar irlandés Alejandro O’Reilly, quien había desempeñado hasta el momento un ejercicio excepcional de control de tropas y recursos militares en la ciudad de La Habana junto al conde de Ricla. ${ }^{5}$ Va a ser el propio militar y político irlandés quien lleve a cabo una primera revisión de las opciones defensivas y tácticas más convenientes para la protección de la nueva provincia. No obstante, estas ideas, estudiadas por Morales Folguera, constituyeron un programa sumamente conservador y falto de innovación. Según el plan, el grueso de las tropas debía permanecer en la principal ciudad de la provincia, Nueva Orleans, mientras que suprimía numerosos puestos militares fronterizos como San Luis de Natchez o Iberbila, a la vez que desaconsejaba la edificación de nuevas construcciones defensivas. ${ }^{6}$ De esta forma, se reforzaba el puesto de la capital en detrimento de otras zonas de interés defensivo, valiosas para contrarrestar acometidas de las numerosas tribus indias que habitaban en la región. Estas decisiones, por otra parte, invitan a pensar en la poca consideración estratégica que se tenía hasta el momento de este paraje, a pesar de la necesidad, como se demostraría en el futuro, de mantener actualizado un enclave costero en el interior del seno mexicano que neutralizase el contrabando mercantil británico o cualquier intento de agresión extranjera.

No será hasta los albores del proceso de independencia americano cuando España vuelva a fijar su atención en Luisiana, gracias fundamentalmente a tres factores. Por un lado, al nombramiento de José de Gálvez como Secretario de Indias y de su sobrino, Bernardo de Gálvez, como gobernador de Luisiana, cuyos trabajos estimularon el impulso que la región necesitaba. Por otro lado, al saneamiento y crecimiento de los fondos económicos destinados a defensa, gracias en parte al adelantamiento de las obras de reestructuración defensiva llevada a cabo en La Habana durante la década anterior y que tantos recursos financieros consumió en su finalización. Por último, la ubicación de este territorio, cuya gestión resultó fundamental para el éxito independentista norteamericano, acaparó la completa atención de la administración española, sabedora de su importancia para frenar las aspiraciones británicas en Florida.

${ }^{4}$ GUTIÉRREZ ESCUDERO, Antonio, "Entre España e Hispanoamérica: Antonio de Ulloa, un hombre de su tiempo. Sus escritos y publicaciones" en LOSADA, Miguel; VARELA, Consuelo (Eds.), Actas del II Centenario de Don Antonio de Ulloa. Sevilla, Consejo Superior de Investigaciones Científicas, CSIC, Escuela de Estudios Hispanoamericanos. 1995, p. 263.

5 KUETHE, Allan J., "Imperativos militares: La Habana, San Juan y la Luisiana en la época de Carlos III" en Historia y Globalización. Ensayos en homenaje a Alfredo Castillero Calvo Panamá, Editora Novo Art S.A., 2017, pp. 68-69.

${ }^{6}$ MORALES FOLGUERA, José Miguel, Arquitectura y urbanismo hispanoamericano en Luisiana y Florida occidental. Málaga, Secretariado de Publicaciones de la Universidad de Málaga, 1987, p. 94. 
A raíz de estas circunstancias, Gálvez delegó en uno de sus hombres de confianza, el militar Francisco de Bouligny, la realización de un detallado informe sobre la situación social de aquella provincia y las medidas más urgentes para su revitalización. ${ }^{7}$ Bouligny, nacido en Alicante en 1736, fue uno de los militares más reconocidos en esta época. Conocedor de los territorios de Luisiana, pues había participado junto con O'Reilly en las tareas de pacificación de 1769, se erigió como la persona idónea para llevar a cabo esta tarea. Su proyecto, presentado en agosto de 1776 y titulado Memoria de la Luisiana, supone uno de los textos más completos y mejor documentados de la situación de esta región durante la segunda mitad del siglo XVIII. ${ }^{8}$ El escrito lo encabeza el siguiente texto:

Noticia con el Estado Actual del comercio y población de la Nueba Orleans, y Luisiana Española, y los medios de adelantar aquella provincia que presenta a Su Majestad Catholica por mano de su Ministro de Indias don Joseph de Gálvez, con el mayor respeto don Francisco Bouligny, capitán del batallón de infantería de aquella provincia. ${ }^{9}$

A lo largo de los once capítulos que componen su Memoria, el alicantino describe detalladamente el escenario de esta provincia, analiza su producción, define a sus habitantes, aborda con detenimiento sus posibilidades comerciales y expone un plan de ampliación poblacional..$^{10}$ No obstante, en los apartados diez y once despliega un programa de fortificaciones y un proyecto económico para sufragarlo, "no solo sin costo del Real Herario, pero con suma ventaja para el país". La décima sección, titulada "Plan de fortificaciones esenciales a aquel país, para su defensa y resguardo", comienza exponiendo cuales son los principales enemigos a tener en consideración y qué parajes son los más vulnerables y necesitados de una revisión defensiva. Según el alicantino, ingleses y tribus indias suponían el mayor peligro en Luisiana, especialmente si aquellos pudiesen aprovecharse del curso de los ríos o de los lagos que circundaban Nueva Orleans para ejecutar sus ataques. Por este motivo, Bouligny centró su discurso en fortalecer el acceso y el tráfico en aquellos cursos fluviales que pusiesen en peligro las principales poblaciones de la región.

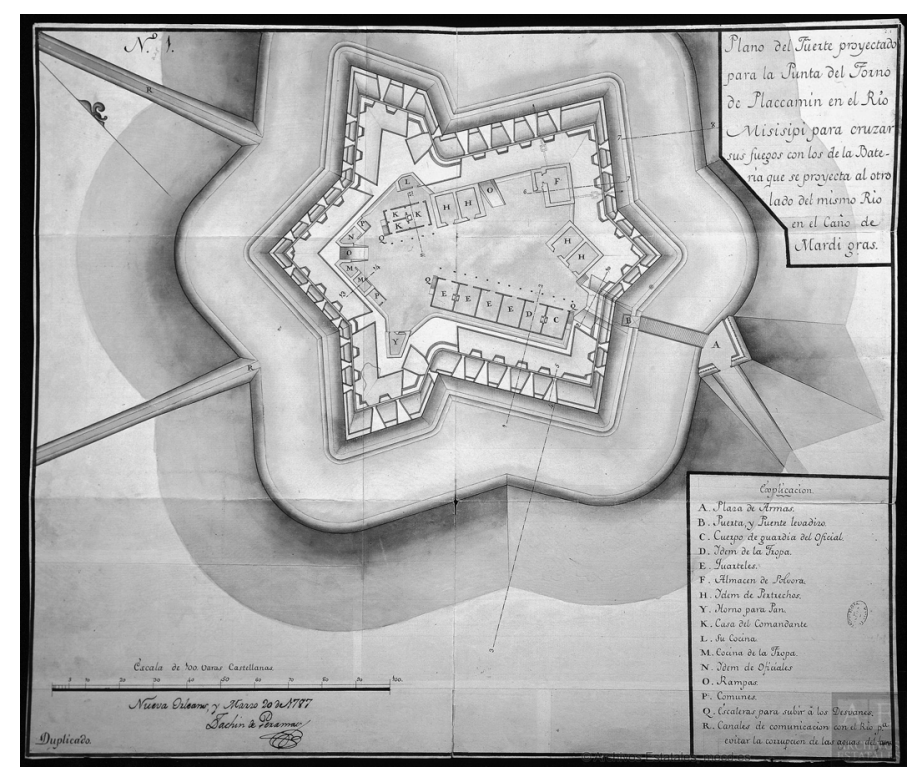

Fig. 1. Plano de la batería proyectada para la entrada del caño de Mardi gras en el río Misisipi, para cruzar sus fuegos con los del fuerte que se proyecta al otro lado del mismo río en la punta del Torno de Placcamin. Joaquín de Peramas. Nueva Orleans, 20 de marzo de 1787. AGI, MP-FLORIDA_LUISIANA, 107.

En primer lugar, propuso la colocación de una fragata de cuarenta cañones del calibre 36 en el río Misisipi, haciendo las veces de batería flotante, "con un costado tan fuerte que ninguna -fragatade los contrarios que pudiese entrar por el río, fuese capaz de ponerse a su lado". Seguidamente, recomendó reforzar el pasaje llamado Torno de los Ingleses, o detour des anglais, previamente fortificado por los franceses, pero cuyas estructuras defensivas habían quedado desmanteladas. En este paraje, distante 15 kilómetros al sur de Nueva Orleans, el río formaba un meandro de difícil navegación, donde las embarcaciones debían detenerse y esperar un cambio de viento para proseguir su ruta. Por ello, planteó la reconstrucción de dos baterías, una a cada lado del Misisipi, prestando especial atención en escoger parajes libres de los habituales desbordamientos del río. Además, apuntaba

\footnotetext{
7 El estudio más completo sobre este personaje se debe a DIN, Gilbert D., Francisco Bouligny: a bourbon soldier in Spanish Louisiana. Nueva Orleans, Louisiana State University Press, 1993.

${ }^{8}$ El informe presentado por el militar alicantino es una de las fuentes principales para conocer la realidad de la Luisiana española en la segunda mitad del siglo XVIII, especialmente para la historiografía americana que ha trabajado sobre este periodo. Por ello, merece traerse a colación la traducción al inglés de su memoria, publicado en: DIN, Gilbert D., Louisiana in 1776. A "memoria" of Francisco Bouligny. Nueva Orleans, Louisiana State University Press, 1977.

9 The Historic New Orleans Collection (De aquí en adelante, THNOC), Bouligny-Baldwin Family Papers, sig. MSS 171, 44, f.1.

10 La memoria ha sido estudiada por: MORALES FOLGUERA, José Miguel, 1987 (6), pp. 94-97.
} 
que ambas estructuras debían estar "bien resguardadas por detrás", con el fin de evitar incursiones por tierra a través de los diferentes bayous o lagunas que existían a poca distancia de aquella zona. ${ }^{11}$ Precisamente con el fin de dificultar el acceso a estas lagunas, el militar español recomendaba reconstruir la pequeña batería localizada en el Bayou de San Juan, sobre el lago Pontchartrain, para

ponerlo al abrigo de todo insulto, pues si todos los ingleses llegasen a poderarse de él, podrían sin el menor imcombeniente remontar por agua las quatro o cinco leguas que ay hasta una poblazion llamada el Bayú, que tenemos distante solo media legua de la ciudad, y como la Nueva Orleans no tiene defenza ahora por la parte de tierra, hazerse dueña de ella a poca costa.

Igualmente, planteó la reconstrucción del fuerte Manchack, cuya estructura se encontraba enormemente debilitada, y la construcción de otro puesto de similares prestaciones en la orilla contraria del río, con el objetivo de cruzar sus fuegos y evitar las correrías de los piratas ingleses. ${ }^{12}$

Otros puntos de la geografía de Luisiana formaron parte de las recomendaciones de Bouligny para su plan. Así, recomendó establecer dos fuertes en Punta Cortada, un paraje situado a más de 400 kilómetros al norte de Nueva Orleans, con el fin de resguardar el río Colorado y evitar la navegación de ingleses o colonos americanos que, en caso de que lograsen la independencia, tuviesen como objetivo el virreinato de Nueva España a través de Texas. Por otra parte, las regiones de Arkansas e Illinoeses, del alto Misisipi, debían ser protegidas con nuevas estructuras defensivas. Por último, consideró imprescindible circundar de murallas la ciudad de Nueva Orleans, tal y como estaba ejecutada en el momento con estacas, colocando un baluarte en cada una de las esquinas de la ciudad.

Todo este programa constructivo, mucho más completo que el presentado varios años antes por
O'Reilly, suponía un cuantioso gasto para las arcas españolas. Por ello, el capítulo undécimo de su Memoria estuvo dedicado a desgranar un plan de pagos que no supusiese un esfuerzo financiero. Según Bouligny, dos casas de comercio españolas con sede en Alicante se ofrecían a proveer a Luisiana una partida de 2.000 esclavos a menor precio que la Real Compañía de Negros de La Habana, hasta el momento la habitual suministradora de esclavos en el entorno caribeño. Los prisioneros deberían ser subastados a los habitantes más poderosos de Luisiana, quienes mediante diversos pagos y algunas retenciones tributarias, podrían sufragar la construcción de las fortificaciones previamente mencionadas sin coste alguno para el erario hispano. No cabe duda que el plan presupuestario presentado por Bouligny se antojaba utópico, a pesar de la convicción que él mismo depositaba en él. ${ }^{13}$

En las conclusiones de su Memoria, el militar alicantino puntualizaba que sus indicaciones debían ser puestas en funcionamiento con la mayor celeridad posible para debilitar definitivamente al ejército británico en territorio norteamericano, pues "hallándose los ingleses, y sus colonias tan distraídas en una Guerra civil que les lleba toda la atención", este se antojaba como el mejor momento para llevar a cabo tales operaciones. Sin embargo, muchas de las observaciones apuntadas por Bouligny no fueron puestas en funcionamiento, y las que sí se tradujeron en proyectos definitivos, tardaron varios años en realizarse.

En este sentido, uno de los ejemplos más notorios fueron las edificaciones llevadas a cabo en el Torno de los Ingleses. Como bien indica Morales Folguera, los reductos Ilamados San Felipe de Placcamin y fuerte Borbón, fueron proyectados en 1787 bajo la gobernación de Esteban Miró, aunque el primero aun tardaría varios años en construirse. ${ }^{14}$ Estas fortificaciones, proyectadas por el ingeniero militar Joaquín de Peramas, ${ }^{15}$ presentaban dos si-

11 THNOC. Bouligny-Baldwin Family Papers, sig. MSS 171, 44, f. 110.

12 El fuerte, construido en 1775 bajo la gobernación de Luis de Unzaga y Amezaga, puede apreciarse en: Archivo General de Indias (De aquí en adelante, AGI) MP-Florida_Luisiana, 75. Plano del fuerte de San Gabriel de Manchac, en Luisiana Manchak, 17 de abril de 1777

13 Según se desprende de su informe, "es tan clara la ventaja de que esta operación resultará al Real Herario que con ella sola se pueden hazer no solo las referidas obras, sino otras de mucha mayor concideracion, poniendo antes de diez años todo aquel país en tal estado de defensa que toda la Inglaterra junta no sea capaz de venirla a insultar". THNOC. Bouligny-Baldwin Family Papers, sig. MSS 171, 44, f. 119.

14 MORALES FOLGUERA, José Miguel (6), pp. 136-137.

15 Para más información sobre este ingeniero, pueden consultarse: CAPEL, Horacio [et. al.], Los Ingenieros militares en España, siglo XVIII: repertorio biográfico e inventario de su labor científica y espacial. Barcelona, Publicacions i edicions de la Universitat de Barcelona, 1983, p. 369. CRUZ FREIRE, Pedro, "Joaquín de Peramas. Un ingeniero militar en América" en LÓPEZ CALDERÓN, Carme; FERNÁNDEZ VALLE, María de los Ángeles y RODRíGUEZ MOYA, María Inmaculada (Coords.), Barroco Iberoamericano: identidades culturales de un imperio. Vol. 1. Santiago de Compostela, Andavira Editora, 2013, pp. 378-379. 
luetas completamente diferentes pero complementarias entre sí. Por un lado, el fuerte Borbón se proyectó como una batería irregular compuesta de tierra y madera, rodeada por un amplio foso y glacis exterior. Su interior contaba con cuerpo de guardia, cuarteles, almacén de pólvora y diversas estancias para el alojamiento de la tropa. En el nivel superior, al que se accedía a través de una pequeña rampa, se distribuían las diferentes cureñas para colocar los cinco cañones del calibre seis destinados a su defensa, los cuales podían colocarse bien enfilando hacia el río o al interior. Ahora bien, dentro de su configuración destacan dos elementos que, por su particularidad, hacen de esta obra un exponente militar singular en territorio americano. En primer lugar, sobresale la ubicación de la plaza de armas, colocada sobre la escarpa del foso y no en el interior del reducto, al que se anexiona mediante un puente levadizo. Por otra parte, la colocación de dos canales que controlaban el flujo de agua desde el Misisipi hasta el foso, posibilitando al gobernador de la fortaleza alterar el interior del mismo de acuerdo a las exigencias bélicas de cada situación.

El fuerte de San Felipe de Placcamin también estuvo proyectado desde 1787, como lo demuestra el Plano del fuerte proyectado para la punta del Torno de Placcamin en el río Misisipi... ${ }^{16}$ Ubicado en la otra orilla del Misisipi con el objetivo de cruzar fuegos con el fuerte Borbón, cumplía las mismas funciones que este, aunque su traza fuese totalmente distinta. En este caso, Peramas proyectó un bastión hexagonal de medidas irregulares, con tres baluartes enfilando hacia tierra y otros tantos al Misisipi. En el interior del mismo se distribuían las dependencias para alojamiento de los oficiales, cuarteles, almacenes de pólvora, hornos, cocinas y diversas estancias comunes. No obstante, repite elementos planteados en el fuerte Borbón, como la plaza de armas tras el foso, comunicada al fuerte mediante un puente levadizo, o los canales de transporte de agua hacia el fuerte. A pesar de estar aprobado desde 1787, no comenzó a construirse hasta 1792, bajo la dirección del ingeniero Gilberto Antonio de San Maxent y el gobierno del XV Barón de Carondelet, dos de las personalidades decisivas en la planificación defensiva de Luisiana en la última década de siglo.

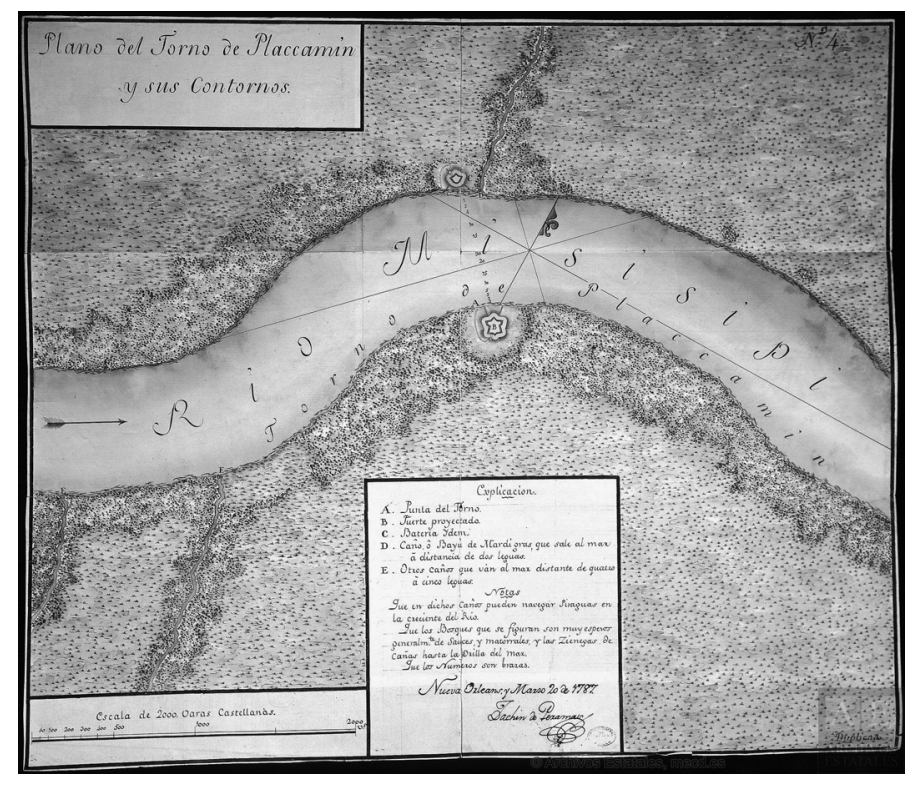

Fig. 2. Plano del Torno de Placcamin y sus contornos. Joaquín de Peramas. Nueva Orleans, 20 de marzo de 1787. AGI, MP-FLORIDA_LUISIANA, 109.

Solo un mes antes de la redacción de la Memoria de Bouligny, la declaración de independencia de los Estados Unidos, efectiva a partir del reconocimiento que Francia hizo de la misma el 6 de febrero de 1778, modificaba enormemente el panorama político, social y territorial americano. Dicho acontecimiento afectó directamente a las posesiones españolas, con especial atención a Luisiana, pasando de ser un estado casi olvidado por la administración española a convertirse en un puesto de primer orden para la conservación de los intereses comerciales y territoriales en el nuevo continente. La emergente nación norteamericana, aliada de España durante las operaciones comandadas por Bernardo de Gálvez en la alta Luisiana y Florida entre 1779 y 1781, pronto se postulaba, no como un aliado, sino como una potencia mundial asentada en sus fronteras. ${ }^{17} \mathrm{~A}$ las premonitorias, y de sobra conocidas, palabras del conde de Aranda sobre los peligros que podría ocasionar Estados Unidos, $^{18}$ hay que sumarle diferentes considera-

\footnotetext{
${ }^{16}$ AGI, MP- Florida Luisiana, 106. Plano del fuerte proyectado, para la punta del torno de Placcamin en el rio Missisipi, para cruzar sus fuegos con los de la batería que se proyecta al otro lado del mismo rio, en el caño de Mardigras. Joaquín de Peramas. Nueva Orleans, 20 de marzo de 1787.

17 SEGURA GARCÍA, Germán, "Operaciones militares en la Alta Luisiana durante la revolución americana" en Bernardo de Gálvez. La presencia de España en México y Estados Unidos. Madrid, Ministerio de Defensa, 2015, pp. 238-239.

18 BEERMAN, Eric, España y la Independencia de Estados Unidos. Madrid, Editorial Mapfre, 1992, p. 27.
}

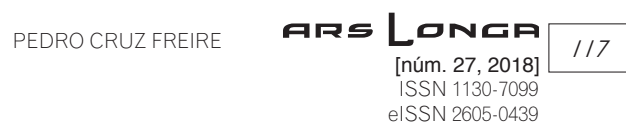


ciones realizadas por otros clarividentes personajes de la esfera política y cultural del momento, caso del cirujano, marino y escritor Pedro Gatell. ${ }^{19}$ En 1780, Gatell redactó la Memoria de la que se demuestra la absoluta necesidad en que se halla la corte de España de poblar y fortificar la Luisiana, especialmente lo correspondiente a las riberas de los ríos Misisipi, Misuri y el de San Bernardo, y las grandes ventajas y utilidades que resultan de tan importante execusión. ${ }^{20}$ Su discurso es una prueba más de la inquietud y la enorme preocupación instalada en torno a la nación americana:

¿Quién creyera que esta república con unos principios tan débiles había de estender sus pabellones hasta en las regiones más remotas del Globo en poco más de un siglo? ¿Quién pensará que havia de ser apetecida para aliada de las Potencias más fuertes? En fin, ¿Quién diría en aquel entonces, que establecida en un suelo tan esteril havia de ser, como la vemos, el almacén del mundo? Pues si sobre tan flacas simientas se ha herigido una potencia capás de disputarle a Roma y Cartago su conducta en materias de Estado y Gobierno, ¿Qué hemos de esperar del nuebo Congreso del Norte de América, rico por la fertilidad de su terreno y orgulloso viéndose competidor con ventaja de la nación reputada hasta el día por guerra y dueña de todos los mares?21

Para el marino español, una sólida defensa de los territorios de Luisiana podría contrarrestar posibles deseos de expansión territorial, cuyos objetivos podrían centrarse no solo en la propia Luisiana, sino también en el virreinato de Nueva España. En su opinión, existían tres puntos clave para mantener a la otrora provincia francesa como antemural del vecino virreinato. En primer lugar, declaraba que fortalecer algunos puntos del río Misuri no solo resultaría beneficioso para facilitar el tráfico en el interior de la provincia, sino también para controlar incursiones de británicos desde Canadá y americanos desde Virginia, asegurando las confluencias de los ríos Ontario, Flineo, y el lago Superior. En segundo lugar, el río San Bernardo, cercano a los dominios mexicanos, conectaba cerca de su desembocadura en el Golfo de México con un afluente que transcurría desde el interior del virreinato. Según Gatell, cualquier enemigo que intentase penetrar por tierra en el Reino de México debía servirse de este cauce, por lo que aconsejaba guarnecer los puntos más vulnerables de su curso. Por último, aunque no hace mención alguna a fortificaciones o puestos defensivos en la ribera del Misisipi, si lo cataloga como un motor y generador de riquezas para la provincia y la propia corona española, por lo que su cuidado permitiría afrontar económicamente las propuestas de su memoria.

En un territorio interior de tanta amplitud y con una acotada línea de costa como Luisiana, la navegación fluvial adquiría una enorme trascendencia. De esta forma se explica la preocupación de Gatell por mantener bien guarnecidos los principales cauces de aquella región, sobre todo si se tiene en consideración que desde la Paz de París de 1763, Gran Bretaña era libre de navegar por el Misisipi con fines comerciales. Si bien este acuerdo parecía agotarse una vez España había derrotado a los británicos en Florida y fuese reconocida la Independencia de las antiguas Trece Colonias mediante el Tratado de Versalles de 1783, la cuestión fluvial se agravó tras estos años. Tal y como indica Andreu Ocariz, Inglaterra, "en un acto de refinada venganza", otorgó a Estados Unidos el derecho a la libre navegación en el Misisipi. ${ }^{22}$ Un contratiempo que España había tratado de superar durante el proceso de independencia americano, cuando Benjamin Franklin intentó negociar con el conde de Aranda la asistencia en la toma de San Miguel de Panzacola a cambio de este derecho, si bien fue rechazado. ${ }^{23}$ Sin embargo, a partir de 1785 la administración española tuvo que lidiar contra esta adversidad, por lo que la atención depositada sobre los ríos durante los próximos años se reforzaría de manera significativa, viéndose reflejada en los planes de defensa ejecutados durante la siguiente década.

Los más importantes proyectos defensivos planteados para Luisiana se elaboraron bajo la gobernación de Luis Francisco Héctor de Carondelet, XV Barón de Carondelet (1791-1797), quien había sucedido a Esteban Miró en la administración de la provincia. El Barón de Carondelet, nacido en Cam-

\footnotetext{
19 Para más información sobre Pedro Gatell, puede consultarse: PESET, José Luis, "La melancolía en la obra de Pedro Gatell, cirujano y marino ilustrado" en Asclepio, 66 (1), 2014, p. 33.

20 SERRANO Y SANZ, Manuel (Ed.), Documentos históricos de la Florida y Luisiana, siglos XVI al XVIII. Madrid, Librería General de Victoriano Suarez, 1912, pp. 353-361.

21 Ibidem, p. 353.

22 ANDREU OCARIZ, Juan José, Luisiana Española. Zaragoza, Talleres Editoriales Librería General, 1975, p. 47.

23 ARNAL SIMÓN, Luis, Arquitectura y urbanismo del septentrión novohispano II. Fundaciones en la Florida y el seno mexicano, siglos XVI al XVIII. México, Universidad Nacional Autónoma de México-Facultad de Arquitectura, 2006, p. 299.
} 
brai en 1747 , fue uno de los militares y políticos españoles más laureados durante la segunda mitad del siglo XVIII. Antes de tomar las riendas de la administración de Luisiana, sirvió como caballero de la Orden de Malta en la Guerra de los Siete Años, asistió al sitio de Argel en 1775 y a la toma de Panzacola en 1781. Como político, fue nombrado gobernador de la provincia de San Salvador desde 1789 a 1791, año en que se le asigna la gobernación de Luisiana y Florida. ${ }^{24}$

Durante su mandato, Carondelet tuvo que enfrentarse a numerosas adversidades políticas y sociales que afectaron al correcto desarrollo de su administración. Entre ellas, las consecuencias de la revolución francesa, la invasión gala en el noreste de España, tensiones territoriales en la frontera con Estados Unidos o el incendio de 1794 que arrasó buena parte del sector suroeste de la ciudad de Nueva Orleans. No obstante, supo responder a estos contratiempos con una política ejemplar alabada por la historiografía.

Lógicamente, las desavenencias con Francia en Europa propiciaron que España se enfrentara a numerosos desafíos, especialmente en los límites geográficos de Luisiana. Por una parte, las graves consecuencias internas desarrolladas en la propia provincia, donde el sentimiento francés aún no había sido abolido completamente. Por otra parte, la amenaza de Inglaterra por mar y desde Canadá. Todo ello sin olvidar la incómoda presencia norteamericana en la periferia de la región. Como producto de estas presiones, el Barón planteó una serie de medidas defensivas que permitieron a la actual región norteamericana afrontar cualquier intento de sublevación interior o asalto por parte de Estados Unidos o Gran Bretaña. Tal y como apunta Morales Folguera, a Carondelet se le debe la ejecución de todo el complejo arquitectónico militar de Nueva Orleans, el impulso de las obras en el Torno de los Ingleses, diversas baterías en el lago Pontchartrain y la actualización de los sistemas defensivos de San Miguel de Panzacola, Mobila, Natchez, Nogales, Manchack, San Marcos de Apalache y otros puntos estratégicos de la zona. ${ }^{25}$ Para ello, se sirvió de la opinión de quienes mejor interpretaban las necesidades defensivas de la provincia, es decir,

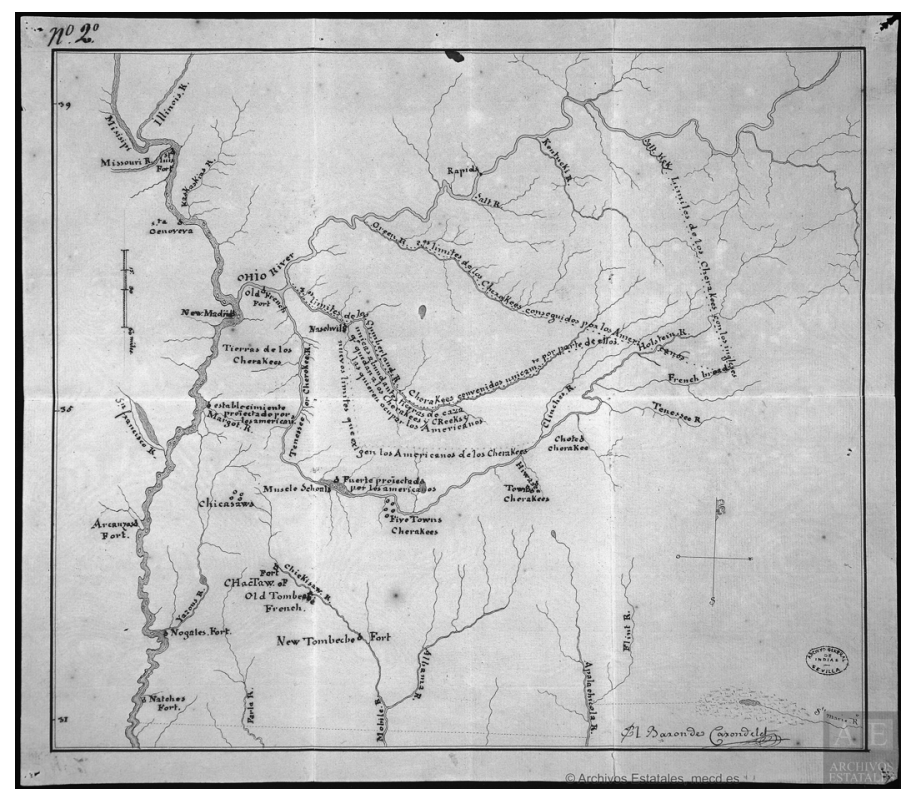

Fig. 3. Mapa de los límites de los Cherokees con los ingleses y de los conseguidos y pretendidos por los norteamericanos en la vertiente oriental de la cuenta del Mississippi. AGI, MP-FLORIDA_LUISIANA, 152. Barón de Carondelet. Nueva Orleans, 1793.

los ingenieros militares. En un periodo de dos años (1792-1794), se reciben otros tantos informes de estos profesionales, en el que detallan las líneas de actuación necesarias para asegurar bajo dominio español aquel territorio.

El primero de estos planes se debe al arquitecto de la corte e ingeniero militar Francisco Sabatini. ${ }^{26}$ Su propuesta, ${ }^{27}$ redactada el 15 de agosto de 1794, comenzaba con una descripción del territorio de Luisiana y un análisis de los posibles escenarios en los que la región podría esperar un ataque. Según el ingeniero, existían tres puntos viables donde podría ejecutarse un asalto, bien mediante un desembarco en la costa en el entorno del Golfo de México, bien por tierra o bien a través de la navegación del río Misisipi. No obstante, el ingeniero italiano se mostraba confiado en que ninguna de

\footnotetext{
24 BEERMAN, Eric, "XV Barón de Carondelet, Gobernador de la Luisiana y la Florida (1791-1797)", en Hidalguía, Gráficas Uguina, 1978, pp. 6-7.

25 MORALES FOLGUERA, José Miguel (6), pp. 98-105.

${ }^{26}$ Un estudio sobre el proyecto defensivo de Francisco Sabatini puede encontrarse en: CABRERA, Leoncio, "Francisco Sabatini y la Fortificación de Luisiana", en Trabajos y Conferencias. Madrid, Facultad de Filosofía y Letras, Universidad de Madrid, II, 1958, pp. 142-149.

27 Archivo Histórico Militar de Madrid. (De aquí en adelante, AHMM). Índice General de Documentos, leg. 5-1-7-7. S/F.
} 
estas opciones pudiera ocasionar daños significativos a la provincia. En primer lugar, descartaba un ataque por mar debido a la imponente presencia española en esta región. Las defensas establecidas en Nueva Orleans, Mobila, San Miguel de Panzacola e hipotéticos refuerzos desde La Habana dificultarían enormemente esta empresa.

Si se hiciese el ataque por medio de un desembarco en la Costa, además de hallarse sus principales puntos como son Panzacola, la Movila y Nueva Orleans cercanos a ella y en tal disposición que pueden ser bien defendidos, se agrega la proporción de que los entren socorros así de los establecimientos que tiene el Rey en el seno mexicano, como de las islas de barlovento, y con especialidad de la Havana.

Si Sabatini creía improbable un acercamiento marítimo, igualmente consideraba un ataque por tierra como una opción de remotas posibilidades. La única potencia preparada para este tipo de asalto sería Estados Unidos, pero esta era una nación carente de disciplina táctica y de recursos ofensivos para efectuar una operación de tanta envergadura. Por ello, juzgaba este intento como "impracticable, aun quando fuese auxiliada de los mencionados indios que habitan el país". Finalmente, la opción del río Misisipi también parecía una empresa de difícil ejecución, dada las pocas facilidades que ofrecía el cauce para transportar artillería, víveres y demás preparativos militares.

Sin embargo, Sabatini recelaba de la nueva nación. Aunque expresaba que las antiguas colonias no estaban preparadas en aquel momento para ejecutar planes ofensivos contra Luisiana y Florida, remarcaba que "es indispensable precautelarse para todo evento, haciendo un plan de defensa que ponga a cubierto dicho establecimiento de la Luisiana y las demás posesiones del Seno Mexicano". Para llevar a cabo tal proyecto, Sabatini planteaba la ejecución de un examen de las distintas plazas y puestos defensivos de Luisiana, con especial atención a Nueva Orleans y Mobila, las dos ciudades mejor posicionadas para proteger la provincia por mar o el Misisipi, depositar los pertrechos de guerra y víveres suficientes durante un ataque y recibir socorros desde La Habana. Igualmente aconsejaba examinar los fuertes de Natchez, Placa- minas y demás puestos avanzados en el interior de la provincia, especialmente los que custodiasen el tráfico fluvial entre diferentes regiones. El estudio de todos estos puestos debía ir acompañado por una serie de planos y perfiles donde se detallasen las mejoras necesarias para cada una de las fortificaciones, así como la ejecución de un mapa en el que se especificasen las distancias entre las ciudades anteriormente mencionadas, los cauces de los principales ríos y la frontera norteamericana. Conjuntamente a estas indicaciones, recomendaba desarrollar una política de acercamiento con los indios pobladores de la provincia, a los cuales consideraba como aliados beneficiosos contra la joven nación norteamericana. ${ }^{28}$

La última parte de su informe la dedicó a ratificar lo aprobado por Junta de Generales el 10 de octubre de 1787, en relación a las fortificaciones de San Miguel de Panzacola, cuya propuesta defensiva fue diseñada por el ingeniero Joaquín de Peramas. ${ }^{29}$ Al ser esta plaza el principal punto defensivo de Florida junto con San Agustín, Sabatini refrendó la decisión de continuar con la construcción del fuerte de San Carlos de Barrancas y la batería baja de San Antonio, así como el fuerte de Santa Rosa, al otro lado de la bahía, para que ambas estructuras cruzasen fuegos en el acceso a la rada norteamericana:

que se lleve a debido efecto la construcción del fuerte de San Carlos y su batería baja de San Antonio, según se proyectó con la sola diferencia que se omita el camino cubierto de dichos fuerte de San Carlos como lo está en San Antonio dejando las dos medias plazas de armas de los flancos: también debe establecerse el fuerte de Santa Rosa en la punta de Sigüenza. ${ }^{30}$

Paralelamente al informe de Sabatini, redactado desde Madrid en 1794, se presentó en Luisiana uno de los textos más detallados y completos de esta década sobre la situación defensiva de Nueva Orleans, producto del trabajo del ingeniero militar Juan María Perchet. Existe muy poca información disponible sobre este personaje, aunque se conoce su participación en la remodelación defensiva de Nueva Orleans durante la década de 1790, además de en otras zonas de la región, pues hay estudios que lo sitúan proyectando el fuerte de

${ }^{28}$ Conseguir el favor de las tribus indígenas fue una constante dentro de las políticas de expansión territorial española en América durante la Edad Moderna. En este aspecto, los ingenieros militares también desarrollaron un papel decisivo. Prueba de ello, puede consultarse: GÁMEZ CASADO, Manuel, "La pacificación de la Guajira por el ingeniero Antonio de Arévalo. Sobre el proyecto de defensa de Sabana del Valle", en Laboratorio de Arte, n²0, 2016, pp. 373-386.

${ }^{29}$ Para más información sobre el proyecto de defensa de San Miguel de Panzacola tras su recuperación en 1781, puede consultarse: CRUZ FREIRE, Pedro (15), pp. 375-388.

${ }^{30}$ AHMM. Índice General de Documentos, leg. 5-1-7-7. S/F. 
San Fernando de Barrancas, en el actual estado de Tennessee. ${ }^{31}$ Dentro de su trabajo como ingeniero ayudante en el Nuevo Conteniente, su Relación de la fortificación de la Plaza de Nueva Orleans en el año de 1794, documento inédito hasta el momento, sintetiza de manera excepcional el estado de los diferentes puestos defensivos de aquella plaza y las condiciones estratégicas que propiciaron sus construcciones. ${ }^{32}$

La descripción geográfica de la capital copa las primeras líneas de su discurso, en el que se desarrolla significativamente un apartado dedicado al ciprés, cuya madera cobró enorme importancia para la arquitectura local por su "buena calidad para construcción, aventajándose en esto al mejor pino, en ser más consistente para obras debaxo del agua, en la qual dura muchísimos años sin corromperse". ${ }^{33}$ A continuación, un halago a la labor de fomento arquitectónico llevado a cabo por el Barón de Carondelet le sirvió para introducir las modificaciones defensivas ejecutadas en ese momento. En primer lugar, aludía a la construcción del circuito amurallado de la ciudad, el cual ya había sido planteado previamente por Francisco Bouligny en la década de 1770 y que finalmente se construye bajo la dirección de Gilberto Guillemard a partir de 1792.

Indicaba Perchet que "por los años de 1791, se hallaba esta plaza abierta por todas partes, expuesta a los insultos de los indios o de qualquiera otra nación que quisiese hacerse dueña de esta colonia". No obstante, temores a un pronto enfrentamiento propiciaron la construcción de una línea defensiva conectada por medio de cinco reductos, llamados de San Carlos, San Juan, San Felipe de Borgoña, San Fernando y San Luis, cuyo objetivo no era otro que resguardar el núcleo poblacional de la ciudad. Además, este cinturón pétreo es un ejemplo extraordinario dentro del contexto americano, pues fue concebido no solo como última línea defensiva ante cualquier enemigo invasor, sino también como primera defensa ante la posibilidad de un ataque perpetrado por los propios habitantes de la ciudad. Una medida razonada atendiendo al pasado francés de la provincia y la vigente declaración de guerra entre ambas naciones. Por esa razón, afirmaba Perchet, se aumentó la proporción del reducto de San Carlos, "colocan-

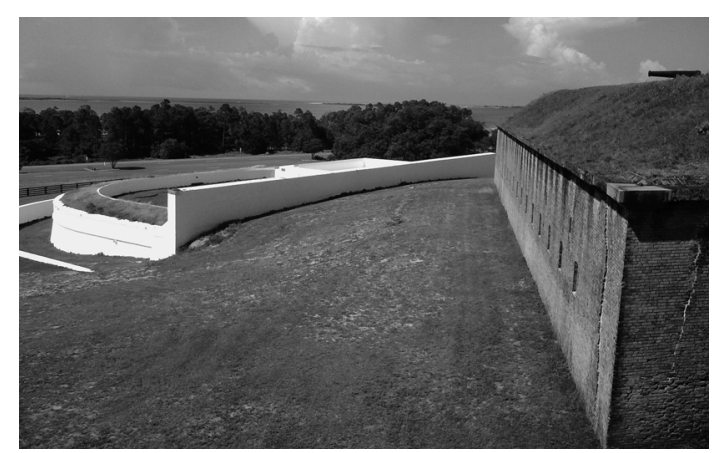

Fig. 4. La batería de San Antonio en Pensacola. Fotografía: autor.

do además sobre su camino cubierto algunas piezas de grueso calibre y un puente levadizo sobre el foso, con la mira de hacerse fuerte en el caso de infidelidad de parte de los vecinos". ${ }^{34}$ Además, las cortinas de unión entre los reductos poseían camino cubierto, estacada y glacis.

Posteriormente, el ingeniero pasa a describir formalmente cada uno de estos reductos, con especial atención al de San Carlos.

Consta de unos lados igualmente que los demás, contiene dentro un capaz repuesto de pólvora, y otro sobre el camino cubierto, alojamiento para la $1^{\circ}$ compañía de granaderos del regimiento fixo de Luisiaina y cuerpo de guardia con un tinglado bajo el que se conservaría efectos de artillería. Tiene montados 32 cañones a saver 5 del calibre de a 30, 1 del de a 27, 2 de bronze del calibre de a 24 y 3 del mismo calibre de a 18 y 3 del de a 8 dentro del reducto y 2 morteros contra la ciudad.

Por su parte, el reducto de San Juan contenía en su interior un cuerpo de guardia y un repuesto de pólvora colocado sobre el glacis, con una artillería montada de los calibres 12, 8 y 4, las mismas características que los de San Felipe de Borgoña y San Fernando. Por último, indicaba que el de San Luis "es el menos capaz, y el más irregular", debido fundamentalmente a los problemas que ocasionaba el terreno donde se fundaron sus cimientos, poco sólido y de mala calidad.

Junto con estos reductos, la defensa de la ciudad se amplió con una batería encarando el río, deno-

\footnotetext{
${ }^{31}$ CAPEL, Horacio [et. al.] (15), pp. 369-370. PATRICK, James, Architecture in Tennessee: 1768-1897. Knoxville, The University of Tennessee Press, 1981, p. 77.

32 THNOC, Sig. MF92, reel 2. Account of the fortification of the plaza of New Orleans. By Perchet. 1794. S/F.

$33 \mathrm{ldem}$.

${ }^{34}$ Idem.
} 


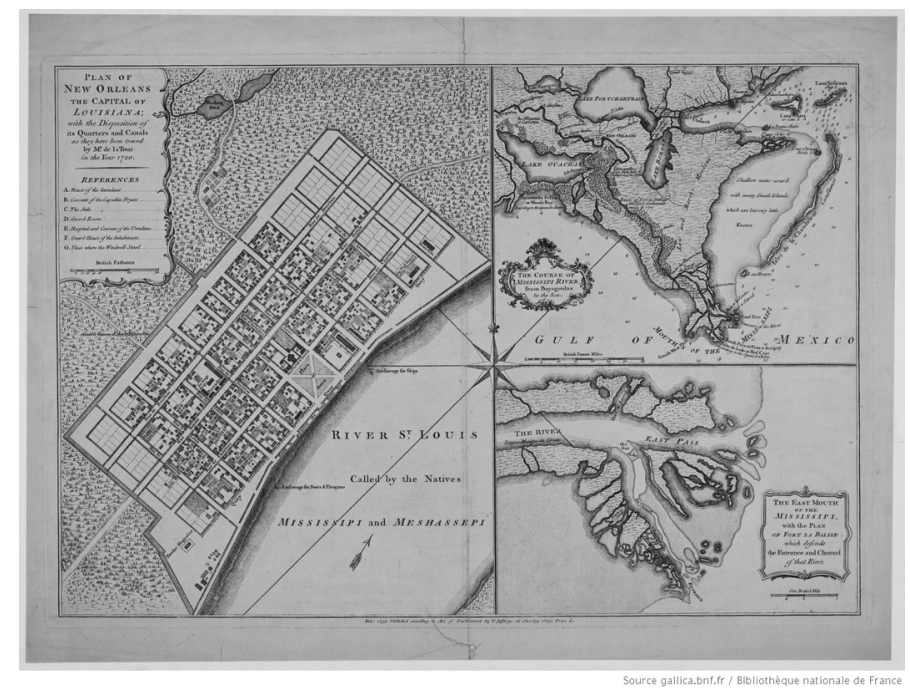

Fig. 5. Cours du fleuve Saint Louis, depuis ses embouchures jusqu'à la rivière d'Iberville et costes voisines. Jacques-Nicolas Bellin Collection d'Anville, Département Cartes et plans, CPL GE DD-2987, sig. 8820, 1764

minada de San Francisco. Construida en piedra y fajina y recubierta en posteriores reparaciones con tablones de madera, preocupaba al ingeniero por su poca consistencia y durabilidad, "pues el mismo fuego que hacen en las salvas los desencajan y desclavan".

Por otra parte, es obligatorio subrayar el esfuerzo con el que Perchet describe las dificultades presentadas a la hora de construir aquel perímetro amurallado. Debido a las características pantanosas del terreno que todavía circunda la ciudad, edificarlo fue únicamente posible gracias al infatigable trabajo de la gobernación, ingenieros y mano de obra empleada en aquella empresa. Por ello, instaba a un continuo cuidado de las instalaciones, pues solo así "podrán mantener algún poco tiempo en pie estos reductos".

Al igual que en la memoria de Bouligny o el informe de Sabatini, Perchet también incluyó en su discurso una relación de las principales vías o puntos por donde podría ser atacada la ciudad. Para analizar las diferentes opciones de ataque, el ingeniero se apoyó en una copia que hizo del mapa ejecutado por el ingeniero francés Jacques-Nicolas BeIlin, titulado Cours du fleuve Saint Louis, depuis ses embouchures jusqu'à la rivière d'lberville et costes voisines y que hoy se conserva en la Biblioteca Nacional de París. ${ }^{35}$ Según su estudio, la primera de las posibilidades sería una incursión desde el seno mexicano a través de la desembocadura del río Misisipi. Sin embargo, la dificultad de cruzar el curso fluvial a contracorriente y el apoyo defensivo instalado en el Torno de los Ingleses dificultaban enormemente esta posibilidad. Una segunda opción consistiría en un ataque desde el lago Pontchartrain y el lago Borque, tal y como advirtieron los informes precedentes. En este sentido, alertaba de la posibilidad de que cualquier escuadra pudiese instalarse en una bahía tras la isla llamada de los Navíos, en las inmediaciones del lago Borque, desde donde podrían conducir sus tropas sin ser vistos y penetrar por el norte de la ciudad. Una tercera vía se planteaba también desde el lago Pontchartrain, a través de un estero navegable que estaba tímidamente defendido por la batería de San Juan. Sin embargo, esta batería, realizada en madera y cerrada con una estacada, podía ser asaltada fácilmente por la espalda dados los cimientos tan pobres de su fábrica. Por estos motivos, y dado que aquel fuerte era "una defensa tan necesaria" recomendaba construir un fuerte de mampostería, en tanto en cuanto consideraba a aquel puesto como "la llave de esta plaza".

Para finalizar el informe, el ingeniero elaboró un capítulo relativo a las ventajas y desventajas de las fortificaciones de Nueva Orleans. En él, trató exclusivamente las características del terreno donde se fundaba la ciudad. Por una parte, admitía que edificar y mantener los diferentes puestos defensivos de la ciudad resultaba ser un arduo trabajo que dependía de una constante preocupación y un considerable desembolso económico. No obstante, este inconveniente se volvía a favor cuando era el enemigo quien debía realizar un ataque.

Los pantanos que la circundan con las cipreras y bosques que al tiro de cañón la rodean, favorecerían mui poco a los ataques del enemigo. Situado en un terreno orizontal y descubierto por todas partes, con dificultad executaria su aproche, si muchos imcombenientes debe renunciar a abrir trinchera en suelo de tal calidad que excavando en quailquier parage, a 3 pies de profundidad, a menos de 24 horas, encontrara sus trincheras llenas de agua. ${ }^{36}$

Asimismo, exponía que la conducción de artillería sobre tal superficie limitaría enormemente el progreso del enemigo, lo que podía ser aprovechado

\footnotetext{
35 Biblioteca Nacional de París (de aquí en adelante, BNP). Cours du fleuve Saint Louis, depuis ses embouchures jusqu'à la rivière d'Iberville et costes voisines. Collection d'Anville, Département Cartes et plans, CPL GE DD-2987 sig. 8820. 1764.

36 THNOC, Sig. MF92, reel 2. Account of the fortification of the plaza of New Orleans. By Perchet. 1794. S/F.
} 
por las milicias de la ciudad que, conocedoras de los terrenos circundantes, tendrían ventaja estratégica para emboscar y repeler cualquier ataque.

Por otra parte, alababa la edificación del reducto de San Carlos, al que consideraba como el más completo de todos los fuertes de la ciudad. En cambio, creía que un revestimiento de mampostería sería necesario para convertirlo en ciudadela en caso de que los demás puestos hubiesen sido destruidos o tomados por el invasor. Además de ello, consideraba que "si se le hubiese formado contra ella un fuerte capaz de una cortina y dos medios baluartes -es decir, un hornabeque anexo- cubriendo la puerta con un pequeño rebellin o plaza de armas atrincherada dando espacio para ello el terreno que media a las calles y casas de la ciudad", la defensa de este puesto estaría completamente asegurada.

Remataba su discurso con un alegato a favor de la provincia, destacando las capacidades de su cultivo y la importancia del río Misisipi para la defensa, no solo de Luisiana, sino también de los vecinos territorios mexicanos. Por ello advertía, como hicieron otros personajes con anterioridad, que la nación norteamericana era consciente de las ventajas que ofrecía este paraje y de la posibilidad de que en el futuro se convirtieran en un enemigo a tener en consideración. Todas estas circunstancias no hacían más que reforzar lo planteado por Perchet en cuanto a la necesidad de mantener actualizado de forma constante las defensas de la ciudad. Por ello, finalizaba con lo siguiente:

Las capitales de los Reynos y Provincias parece que son el móvil y agente que govierna lo restante, si estas no se hacen respetables, mal lo serán sus territorios subalternos. ¿Cómo podrá serlo una plaza, cuyas fortificaciones cuesta tanto de mantenerlas en pie aun si haver experimentado los extragos del cañón enemigo? Donde apenas alcanzan las tropas para guarnecer los puestos del rio arriva, tan interesantes quanto de su conservación depende la de la misma plaza en particular los socorros de víveres. ¿Y quién sería más dueño del golfo mexicano que el que lo sea del río Mississipi? ${ }^{37}$

A partir de los datos ofrecidos pueden alcanzarse diversas conclusiones. En primer lugar, parece evidente el esfuerzo y la preocupación constante exhibidas por la monarquía española en estos territorios, un hecho que no ha sido lo suficientemente valorado por la historiografía española. Reforzar defensivamente la Luisiana se convirtió en un apasionante reto para para el gobierno hispano duran- te cuatro décadas, debido a las numerosas confrontaciones territoriales que surgieron entre Francia, España, Inglaterra y la joven nación norteamericana. Todo ello a pesar de numerosos inconvenientes, entre ellos la falta de personal cualificado encargado de supervisar los principales puntos estratégicos de la provincia o la exigua financiación recibida para edificaciones militares. Tales inconvenientes derivaron en una escasa aportación defensiva, tanto en elementos construidos como en la innovación de las propuestas presentadas, donde prácticamente se reproducen los planes ejecutados décadas atrás por los ingenieros militares franceses. Ello se hace visible principalmente en la ejecución del circuito amurallado de la ciudad o en los proyectos para proteger el Torno de los Ingleses olas baterías interiores de los lagos Borque y Pontchartrain, tal y como se ha analizado previamente en este estudio. No cabe duda de que aún quedan pendientes muchas incógnitas que plantear y resolver sobre este tema. Sin embargo, se espera que con el presente estudio se haya podido aclarar las principales medidas de seguridad adoptadas por la corona para mantener bajo su gobierno este valioso territorio en el entorno del seno mexicano.

\section{Bibliografía}

ANDREU OCARIZ, Juan José. Luisiana Española. Zaragoza, Talleres Editoriales Librería General, 1975.

ARNAL SIMÓN, Luis. Arquitectura y urbanismo del septentrión novohispano II. Fundaciones en la Florida y el seno mexicano, siglos XVI al XVIII. México, Universidad Nacional Autónoma de México-Facultad de Arquitectura, 2006.

BEERMAN, Eric. España y la Independencia de Estados Unidos. Madrid, Editorial Mapfre, 1992.

CAPEL, Horacio; GARCIA, Lourdes; MONCADA, José Omar; OLIVE, Francesc; QUESADA, Santiago; RODRIGUEZ, Antonio; SANCHEZ, Joan Eugeni; TELLO, Rosa. Los Ingenieros militares en España, siglo XVIII: repertorio biográfico e inventario de su labor científica $y$ espacial. Barcelona, Publicacions i edicions de la Universitat de Barcelona, 1983.

CRUZ FREIRE, Pedro. "Joaquín de Peramas. Un ingeniero militar en América" en LÓPEZ CALDERÓN, Carme; FERNÁNDEZ VALLE, María de los Ángeles y RODRÍGUEZ MOYA, María Inmaculada (Coords.). Barroco Iberoamericano: identidades culturales de un imperio. Vol. 1. Santiago de Compostela, Andavira Editora, 2013, pp. 375-388.

DEL CANTILLO, Alejandro. Tratados, convenios y declaraciones de Paz y de Comercio que han hecho con las potencias extranjeras los monarcas españoles de la casa de Borbon desde el año 1700 hasta el día. Madrid, imprenta de Alegría y Charlain, 1843.

DIN, Gilbert D. Francisco Bouligny: a bourbon soldier in Spanish Louisiana. Nueva Orleans, Louisiana State University Press, 1993. 
DIN, Gilbert D. Louisiana in 1776. A "memoria" of Francisco Bouligny. Nueva Orleans, Louisiana State University Press, 1977.

GÁMEZ CASADO, Manuel. "La pacificación de la Guajira por el ingeniero Antonio de Arévalo. Sobre el proyecto de defensa de Sabana del Valle", en Laboratorio de Arte, $n^{\circ} 20,2016$, pp. 373-386.

GUTIÉRREZ ESCUDERO, Antonio. “Entre España e Hispanoamérica: Antonio de Ulloa, un hombre de su tiempo. Sus escritos y publicaciones" en LOSADA, Miguel; VARELA, Consuelo (Eds.). Actas del II Centenario de Don Antonio de Ulloa. Sevilla, Consejo Superior de Investigaciones Científicas, CSIC, Escuela de Estudios Hispanoamericanos. 1995, pp. 257-270.

KUETHE, Allan J. "Imperativos militares: La Habana, San Juan y la Luisiana en la época de Carlos III" en Historia y Globalización. Ensayos en homenaje a Alfredo Castillero Calvo Panamá, Editora Novo Art S.A., 2017, pp. 59-80.

MORALES FOLGUERA, José Miguel. Arquitectura y urbanismo hispanoamericano en Luisiana y Florida occi- dental. Málaga, Secretariado de Publicaciones de la Universidad de Málaga, 1987.

PATRICK, James. Architecture in Tennessee: 1768-1897. Knoxville, The University of Tennessee Press, 1981.

PESET, José Luis. "La melancolía en la obra de Pedro Gatell, cirujano y marino ilustrado" en Asclepio, 66 (1), 2014, p. 33.

SAN MIGUEL PÉREZ, Enrique. "La crisis de la estrategia política española en Norteamérica: de Bernardo de Gálvez a Luis de Onís" en MARTíNEZ PEÑAS, Leandro y FERNÁNDEZ RODRÍGUEZ, Manuela (Coords.). EI ejército y la armada en el noroeste de América: Nootka y su tiempo. Madrid, Universidad Rey Juan Carlos, 2012, pp. 29-58.

SEGURA GARCÍA, Germán. "Operaciones militares en la Alta Luisiana durante la revolución americana" en Bernardo de Gálvez. La presencia de España en México y Estados Unidos. Madrid, Ministerio de Defensa, 2015, pp. 235-244.

SERRANO Y SANZ, Manuel (Ed.). Documentos históricos de la Florida y Luisiana, siglos XVI al XVIII. Madrid, Librería General de Victoriano Suarez, 1912. 